DRAFT VERSION MAY 15, 2018

Preprint typeset using LTEX style emulateapj v. 01/23/15

\title{
A GLOBAL ILC APPROACH IN PIXEL SPACE OVER LARGE ANGULAR SCALES OF THE SKY USING CMB COVARIANCE MATRIX
}

\author{
VIPIN SUDEVAN $^{1}$, RAJIB SAHA $^{1}$ \\ Draft version May 15, 2018
}

\begin{abstract}
We propose a new internal linear combination (ILC) method in the pixel space, applicable on large angular scales of the sky, to estimate a foreground minimized Cosmic Microwave Background (CMB) temperature anisotropy map by incorporating prior knowledge about the theoretical CMB covariance matrix. Usual ILC method in pixel space, on the contrary, does not use any information about the underlying CMB covariance matrix. The new approach complements the usual pixel space ILC technique specifically at low multipole region, using global information available from theoretical CMB covariance matrix as well as from the data. Since we apply our method over the large scale on the sky containing low multipoles we perform foreground minimization globally. We apply our methods on low resolution Planck and WMAP foreground contaminated CMB maps and validate the methodology by performing detailed Monte-Carlo simulations. Our cleaned CMB map and its power spectrum have significantly less error than those obtained following usual ILC technique at low resolution that does not use CMB covariance information. Another very important advantage of our method is that the cleaned power spectrum does not have any negative bias at the low multipoles because of effective suppression of CMB-foreground chance correlations on large angular scales of the sky. Our cleaned CMB map and its power spectrum match well with those estimated by other research groups.

Subject headings: cosmic background radiation - cosmology: observations — diffuse radiation
\end{abstract}

\section{INTRODUCTION}

For reconstruction of Cosmic Microwave Background (CMB) signal from multi-frequency observations an important method is Internal-Linear-Combination (ILC) (Tegmark \& Efstathiou 1996; Tegmark et al. 2003; Bennett et al. 2003; Eriksen et al. 2004; Saha et al. 2006; Hinshaw et al. 2007). To obtain a foreground minimized CMB map the ILC method requires neither to explicitly model the frequency spectra of individual foreground components, nor does it require to model the foreground amplitudes (at some reference frequency) in terms of so called foreground templates. The only assumption one makes related to foregrounds is that each of them has a frequency spectrum that is different from the frequency spectrum of CMB component, which is assumed to be that of black-body in nature (Mather et al. 1994; Fixsen et al. 1996). The basic idea behind the ILC method is to linearly superpose the available foreground contaminated CMB maps using certain amplitude terms, a set of weights, to estimate a foreground minimized CMB map. The weights are obtained by minimizing the variance of the cleaned map and can be computed analytically by using a simple formula. In spite of being simple to design and yet a powerful technique to reconstruct a cleaned CMB map we see that it is necessary - for a few important reasons - to investigate performance of the usual ILC method in some hitherto unexplored cases. First, while estimating the weights the usual ILC method in pixel space does not take into account the covariance structure of the CMB maps. In other words, it does not use the fact that the final cleaned map, if perfectly cleaned of all foregrounds and detector noise is negligible, should have a covariance structure consistent with the underlying theoretical model. Secondly, some of the maximum likelihood methods (Eriksen et al. 2007, 2008a,b; Gold et al. 2011; Planck Collaboration

\footnotetext{
${ }^{1}$ Physics Department, Indian Institute of Science Education and Re search Bhopal, Bhopal, M.P, 462066, India.
}

et al. 2016c,a) for component separation however use CMB and detector noise covariance matrices to reconstruct $\mathrm{CMB}$ and all foreground components. It is therefore natural to ask a question can we generalize usual ILC method in pixel space to incorporate $C M B$ covariance information also?

In the present work we seek to find a solution to the above problem and generalize the pixel space ILC method taking into account prior information of the theoretical covariance matrix of the CMB maps. Therefore, instead of minimizing simple variance of the cleaned map we propose to estimate the weights by minimizing the reduced variance of the cleaned map, the reduced variance being defined by the CMB covariance weighted variance of the cleaned map, which is explained in Section 2. Since storage space into the computer disks of such full pixel-space covariance matrix increases rapidly with the HEALPix pixel resolution parameter $N_{\text {side }}\left(\sim N_{\text {side }}^{4}\right)$, in the current work we use low pixel resolution maps. Further to focus largely on the low multipoles we smooth the input $N_{\text {side }}=16$ maps by a Gaussian window function of FWHM $9^{\circ}$. At this smoothing the input maps contain approximately 2.5 pixels per beam width, which implies these maps are properly band-width limited. The larger beam smoothing also reduces detector noise contributions at different pixels.

Our method at low resolution bears an interesting complementarity in its approach when compared with the usual pixel space ILC method at high resolution, that do not use the CMB covariance matrix. Since the level of foreground contamination, and their spectral properties vary with the sky positions, in a high resolution analysis of usual ILC method one performs foreground cleaning individually over several smaller regions of the sky, in such a way that the foreground spectral properties and level of foreground contaminations in each region remains approximately constant. Because of low pixel resolution (and large smoothing on the low pixel resolution maps) of this work we chose either to perform foreground re- 
moval over the entire sky or by dividing the sky into small number of regions. In the second approach we divide the sky into two regions and clean them individually in a total of two iterations. Our aim is to use as much large sky fraction as possible during foreground removal and information about CMB theoretical covariance matrix from the corresponding large fraction, so that our method becomes a global method of foreground minimization. Thus our method may be seen as dual to usual high resolution ILC method, wherein the former uses global information from the covariance matrix and the data to estimate the foreground minimized CMB map and the later relies upon the local information of foregrounds properties.

By performing detailed Monte-Carlo simulations we find that the new ILC method of this work has significantly less reconstruction errors in cleaned maps and power spectrum than the usual ILC method in pixel space over large angular scales of the sky. The cleaned power spectrum of our method does not have a negative bias at the low multipole region that is present in usual ILC method and is caused by a chance correlations between $\mathrm{CMB}$ and foreground components on a particular realization of CMB sky.

The subject of component separation in the context of CMB is very rich. Bunn et al. (1994); Bouchet et al. (1999) propose a Wiener filter approach. Saha et al. (2008) discuss in detail bias issues in CMB angular power spectrum for harmonic space ILC approach. Saha \& Aluri (2016) apply an ILC technique to jointly estimate CMB and foreground components for Stokes Q polarization in presence of varying spectral index of synchrotron component. Iterative harmonic space ILC algorithm was applied on high resolution Planck and WMAP data, and one of its limitations arising due to foreground leakage was first discovered and remedied by Sudevan et al. (2017). Basak \& Delabrouille (2012) and Basak \& Delabrouille (2013) implement a needlet space ILC algorithm to incorporate localization of foreground emissions both in pixel space and its 'Fourier' space. A variant of ILC technique by minimizing a measure of non-Gaussianity was implemented on WMAP temperature and Polarization data by Saha (2011) and Purkayastha \& Saha (2017) respectively. Eriksen et al. (2007, 2008a,b) propose Gibbs sampling for component separation. Gold et al. (2011) use Markov Chain Monte Carlo method to jointly estimate CMB and foregrounds from WMAP data.

We organize our paper as follows. In Section 2 we discuss the formalism of the new method. We describe how to compute the theoretical CMB covariance matrix in Section 3 and comment on its singular nature in Section 4. In Section 5 we describe in detail our foreground minimization approaches on Planck and WMAP low resolution maps. We discuss the cleaned maps and CMB angular power spectra obtained from data on Section 6. We validate our foreground minimization methods by performing Monte Carlo simulations in Section 7. In Section 8 we show the advantage of the new ILC approach in pixel space over the usual ILC approach for analysis over large angular scales on the sky. We investigate the role of CMB-foreground chance correlation in not-so-efficient foreground removal by the usual ILC methods at low resolution in Section 9 and comment that using the CMB covariance matrix in our new method, we effectively suppress such chance correlations which leads to improved foreground minimization. Finally we conclude in Section 10.

\section{FORMALISM}

Let we have $n$ full sky foreground contaminated CMB maps, $\mathbf{X}_{i}$ at a frequency $\nu_{i}$, with $i=1,2, \ldots, n$ at some beam and pixel resolution in thermodynamic temperature unit. We assume mean temperature corresponding to each frequency $\nu_{i}$ has already been subtracted from each $\mathbf{X}_{i}$. $\mathbf{y}$ represents the cleaned CMB map obtained by linear combination of $n$ input maps $\mathbf{X}_{i}$, with weight factor $w_{i}$, i.e.,

$$
\mathbf{y}=\sum_{i=1}^{n} w_{i} \mathbf{X}_{i} .
$$

Here each $\mathbf{X}_{i}$ and $\mathbf{y}$ are $N \times 1$ vectors describing full sky HEALPix ${ }^{2}$ map with $N$ pixels for a pixel resolution parameter $N_{\text {side }}\left(N=12 N_{\text {side }}^{2}\right)$, smoothed by Gaussian beam of certain FWHM. Instead of minimizing cleaned map variance $\mathbf{y}^{T} \mathbf{y}$ like the usual pixel space ILC method we propose a more general approach by incorporating the prior information about the theoretical CMB covariance matrix. We minimize,

$$
\sigma^{2}=\mathbf{y}^{T} \mathbf{C}^{\dagger} \mathbf{y}
$$

where $\mathbf{C}$ represents the $\mathrm{CMB}$ theoretical covariance matrix which as discussed in Section 4 may not be always invertible. $\mathbf{C}^{\dagger}$ represents Moore-Penrose generalized inverse (Moore 1920; Penrose 1955) of matrix C. Using Eqn. 1 we can write Eqn. 2 as

$$
\sigma^{2}=\mathbf{W} \mathbf{A} \mathbf{W}^{T},
$$

where $\mathbf{W}=\left(w_{1}, w_{2}, w_{3}, \ldots, w_{n}\right)$ is a $1 \times n$ row vector of weight factors of different frequency maps and $\mathbf{A}$ is an $n \times n$ matrix with its elements $A_{i j}$ satisfying

$$
A_{i j}=\mathbf{X}_{i}^{T} \mathbf{C}^{\dagger} \mathbf{X}_{j} .
$$

Since spectral distribution of CMB photons is that of a blackbody to a very good approximation, CMB anisotropy in thermodynamic temperature unit is independent on frequency bands. To reconstruct $\mathrm{CMB}$ anisotropies without introducing any multiplicative bias in its amplitude we constrain the weights for all frequency bands to sum to unity, i.e., $\sum_{i=1}^{n} w_{i}=$ 1 . The choice of weights that minimize the variance given by Eqn. 2 is obtained following a Lagrange's multiplier approach (e.g., see Saha et al. (2008) and also Tegmark \& Efstathiou (1996); Tegmark et al. (2003); Saha et al. (2006))

$$
\mathbf{W}=\frac{\mathbf{e A}^{\dagger}}{\mathbf{e A}^{\dagger} \mathbf{e}^{T}},
$$

where $\mathbf{A}^{\dagger}$ represents Moore-Penrose generalized inverse of matrix $\mathbf{A}$ and $\mathbf{e}=(1,1, \ldots, 1)$ is a $1 \times n$ row-vector representing shape vector of $\mathrm{CMB}$ in thermodynamic temperature unit.

\section{COMPUTING CMB COVARIANCE MATRIX}

To compute elements of the CMB covariance matrix, $\mathbf{C}$ we assume principle of statistical isotropy of CMB anisotropy. Under this assumption the elements $C_{i j}$ of matrix $\mathbf{C}$, at the chosen beam and pixel resolution are given by

$$
C_{i j}=\sum_{\ell=2}^{\ell=\ell_{\max }} \frac{2 \ell+1}{4 \pi} C_{\ell} B_{\ell}^{2} \mathcal{P}_{\ell}\left(\cos \left(\gamma_{i j}\right)\right) P_{\ell}^{2},
$$

where $C_{\ell}$ is the fiducial CMB angular power spectrum (Planck Collaboration et al. 2016d), $B_{\ell}$ represents the beam transfer

${ }^{2}$ Hierarchical Equal Area Isolatitude Pixellization of sphere, e.g., see Górski et al. (2005) 

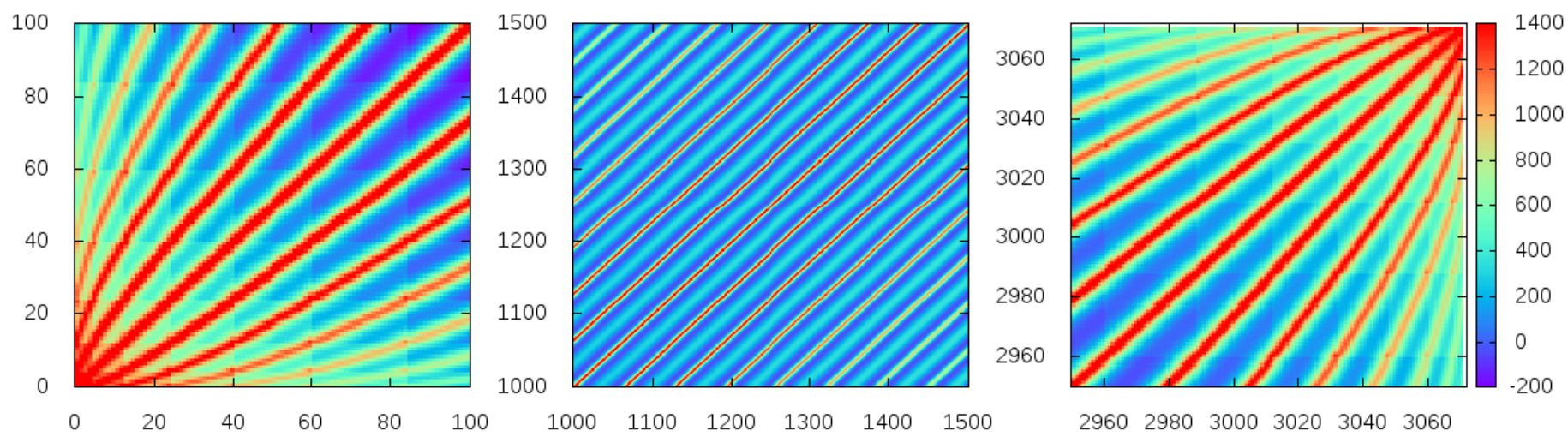

FIG. 1.- Different (square) block matrices taken across the diagonal of the theoretical CMB covariance matrix, C. The non diagonal nature of this matrix is consistent with statistically isotropic CMB. Color map unit is in $\mu K^{2}$ (thermodynamic).

function, $\mathcal{P}_{\ell}$ denote Legendre polynomials and $P_{\ell}$ is pixel window function for the given $N_{\text {side }}$ parameter. The cosine of the angle $\gamma_{i j}$ is obtained following

$$
\cos \left(\gamma_{i j}\right)=\cos \left(\theta_{i}\right) \cos \left(\theta_{j}\right)+\sin \left(\theta_{i}\right) \sin \left(\theta_{j}\right) \cos \left(\phi_{i}-\phi_{j}\right),
$$

where $\left(\theta_{i}, \phi_{i}\right)$ and $\left(\theta_{j}, \phi_{j}\right)$ are spherical polar angles respectively of $i$ and $j$ th pixels of the map. Under the assumption of statistical isotropy $\mathbf{C}$ is independent on any particular choice of coordinate system (e.g., Galactic, Ecliptic, or any Euler rotated version of these coordinate systems) in which the input maps are provided. We note, however, the assumption of statistical isotropy is not a necessity in our method. If needed, we can also use a covariance matrix compatible to statistically anisotropic model which may be caused due to non-trivial primordial power spectrum (Ghosh et al. 2016; Contreras et al. 2017).

\section{IS C SINGULAR?}

As is the case for this work, rank, $r$, of $\mathbf{C}$ is less than its dimension $N$. The rank of $\mathbf{C}$ is simply equal to effective number of independent $a_{\ell m}$ modes (real and imaginary) that are used in Eqn. 6 to generate each element of the theoretical covariance matrix. A quick calculations shows that, $r=\left(\ell_{\max }+1\right)\left(\ell_{\max }+2\right)-\left(\ell_{\max }+1\right)-4$, when the summation over multipoles in Eqn 6 extends upto $\ell=\ell_{\max }$. Since we use, $N_{\text {side }}=16$ HEALPix maps in our analysis, $\ell_{\max }=2 \times N_{\text {side }}=32$ for us, implying $r=1085$ which is less than dimension of $\mathbf{C}$, which is $N=3072$. Since $\mathbf{C}$ is singular we use its generalized inverse in Eqn. 2.

\section{METHODOLOGY}

\subsection{Input maps and Data Processing}

We use Planck 2015 released LFI 30, 44 and 70 GHz, HFI $100,143,217$ and $353 \mathrm{GHz}$ frequency maps along with the WMAP 9 year difference assembly (DA) maps in our analysis. For each of these maps we convert them to spherical harmonic space upto $\ell_{\max }=32$ and smooth the resulting $a_{\ell m}$ coefficients by the ratio $B_{\ell}^{0} P_{\ell}^{0} / B_{\ell}^{i} P_{\ell}^{i}$ where $B_{\ell}^{i}$ and $P_{\ell}^{i}$ represent the beam and pixel window functions of the original maps whereas $B_{\ell}^{0}$ and $P_{\ell}^{0}$ represent the corresponding window functions for the $N_{\text {side }}=16$ maps. We take $B_{\ell}^{0}$ corresponding to a Gaussian beam of FWHM $=9^{\circ}$. We convert the smoothed spherical harmonic coefficients to $N_{\text {side }}=16$ maps using HEALPix supplied facility synfast. For each of WMAP Q, V and $\mathrm{W}$ bands we average all the DA maps for any given frequency band. We convert all these maps in

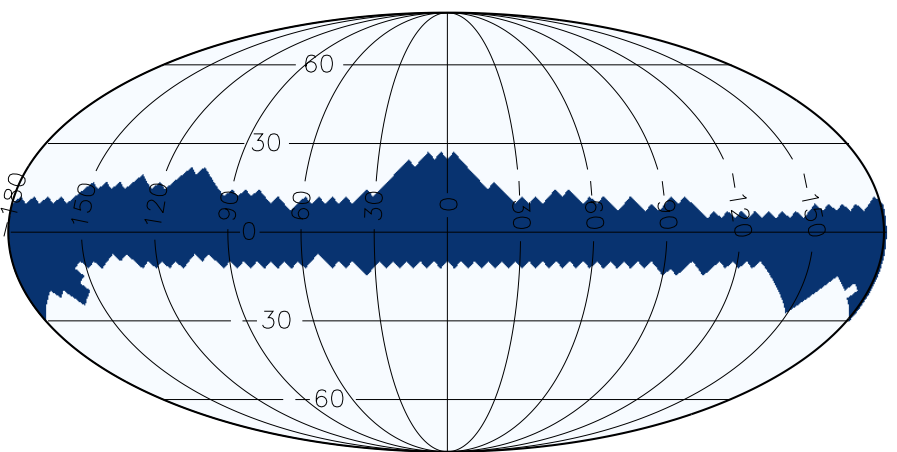

FIG. 2.- The blue region shows sky portions dominated by the strong thermal dust emission and is removed by the ThDust 5000 mask

$\mu K$ (thermodynamic) temperature unit and subtract the corresponding mean temperature from each frequency map. This results in a total of 12 input maps for foreground removal at $N_{\text {side }}=16$.

\subsection{Method-1}

Since we are interested in a global method of foreground minimization our aim is to use as much sky region as possible to estimate the weights. In the first method we therefore estimate the weights using information obtained from the entire sky. We first estimate full sky CMB theoretical covariance matrix using Eqn. 6. We obtain $\mathbf{C}^{\dagger}$ using singular value decomposition of $\mathbf{C}^{\dagger}$ and applying a cutoff of $1.0 \times 10^{-7}$ on the singular values. We show different square blocks across the diagonal of $\mathbf{C}$ matrix estimated for the entire sky in Fig. 1. Non-diagonal elements of this matrix show significant coupling between different pixel pairs for a pure CMB map and justifies using Eqn. 2 for minimization instead of ignoring such correlations as is done in usual pixel space ILC approach. Using $\mathbf{C}^{\dagger}$ we obtain weights for foreground removal using Eqns. 4 and 5. The cleaned map obtained using these weights is discussed in Section 6.

\subsection{Method-2}

Since when compared with the expected level of CMB temperature anisotropy, the region near galactic plane is strongly contaminated by the foregrounds than the outside region, it is desirable to perform foreground removal separately on the sky region away from the plane and inside the plane. Moreover the spectral properties of the foregrounds vary with sky positions, specifically near the galactic plane. WMAP science team produce the internal linear combination map at 


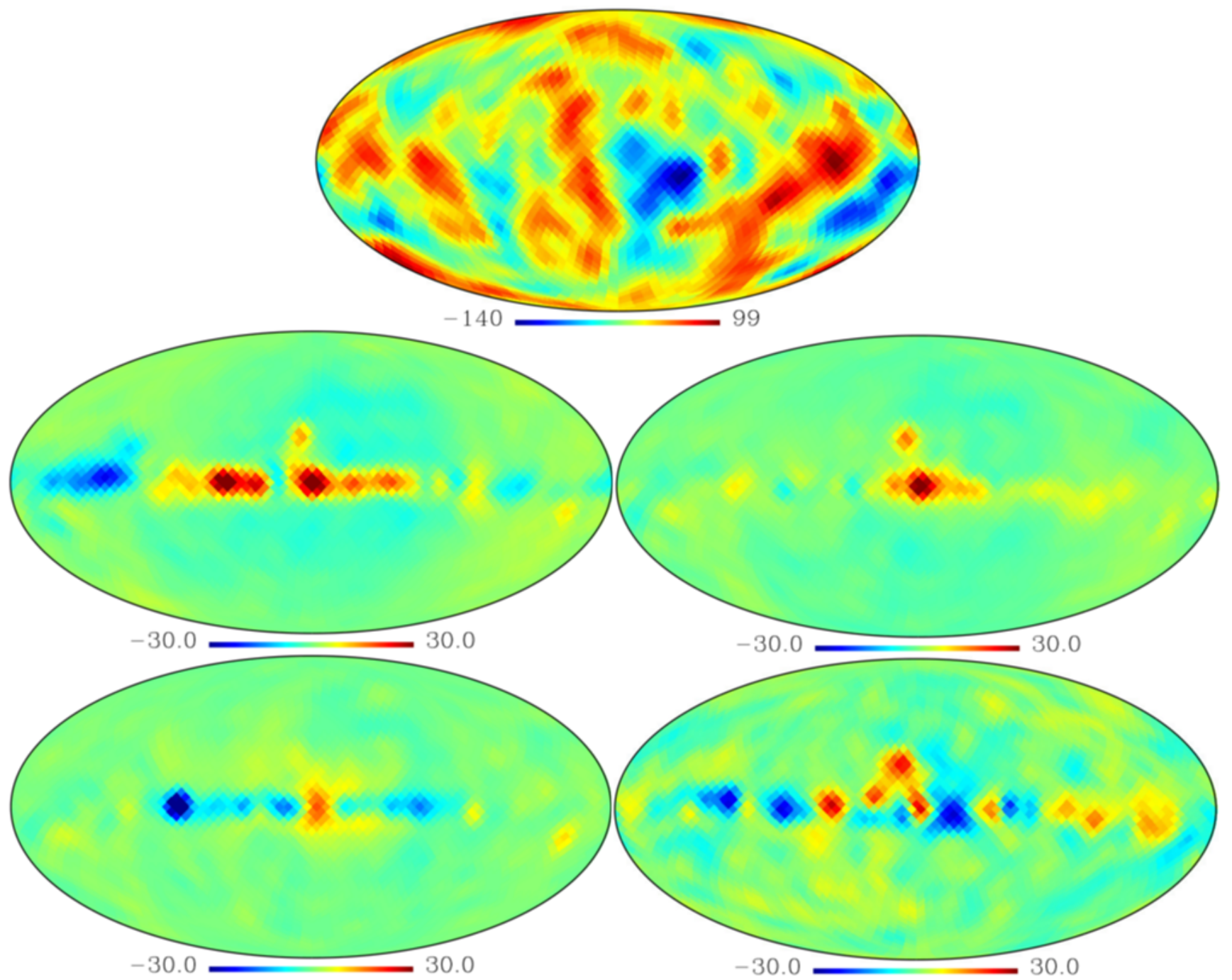

FIG. 3. - Foreground cleaned CMB map (CMap1) obtained following Method- 1 of this work at $N_{\text {side }}=16$ and FWHM $=9^{\circ}$ corresponding to Gaussian beam is shown in the top panel. The middle left and middle right figures show the difference maps COMMANDER - CMap1 and NILC - CMap1 respectively. The bottom panel from left to right show SMICA - CMap1 and WMAP ILC - CMap1. Any residual monopole and dipole have been removed from all difference maps to highlight residuals on cosmologically important scales. All color scales are in $\mu K$ thermodynamic temperature unit.

$N_{\text {side }}=512$ by dividing the galactic plane into 12 different regions. The sky region outside the plane was cleaned in a single iteration. The work of this paper, however, intends to use global information from the theoretical CMB covariance matrix and data. Keeping in mind such dual requirements we divide the sky into two regions and clean each as described below. The reason why we divide the sky into smaller number of regions than an usual ILC approach in pixel space at high resolution, is that we are interested in low resolution maps focusing on the low multipoles. The lack of structures on small scales in the input maps ensures that the sky regions need not be too small.

\subsubsection{Sky Division}

To identify the region near the galactic plane that contains strong foreground emissions we take Planck $353 \mathrm{GHz}$ and 70 $\mathrm{GHz}$ frequency maps at $N_{\text {side }}=2048$. We downgrade these maps to $N_{\text {side }}=256$ and smooth them by the ratio of window functions of a Gaussian beam of FWHM $=6^{\circ}$ and the original beam functions of the $N_{\text {side }}=2048$ maps at the their native resolutions. We subtract resulting reduced resolution $70 \mathrm{GHz}$ map from $353 \mathrm{GHz}$ map at $N_{\text {side }}=256$. The difference map contains strong emissions from thermal dust at $353 \mathrm{GHz}$. We identify pixels of the difference map with values $\geq 5000 \mu \mathrm{K}$ and assign a value of unity to them and zero to rest. We downgrade this binary map at $N_{\text {side }}=16$. Finally we reassign all non-zero pixels of the downgraded map a value of zero and the rest to a value of unity. This sky region defined by the zero pixel values contains strong thermal dust emissions. The region complementary to this strong thermal dust emission is survived after application of the ThDust 5000 mask. The sky region removed by this mask is shown in deep blue color in Fig. 2.

\subsubsection{Foreground cleaning}

Based upon the discussions of the previous sections we perform the foreground cleaning following the second method in following three steps.

1. We estimate the covariance matrix $\tilde{\mathbf{C}}$ applicable for the sky region defined by the ThDust 5000 mask. This is done by using Eqn. 6 for all the pixel pairs $(i, j)$ that survive after application of the mask. We estimate $\tilde{\mathbf{C}}^{\dagger}$ following the same procedure as described in Section 5.2. 


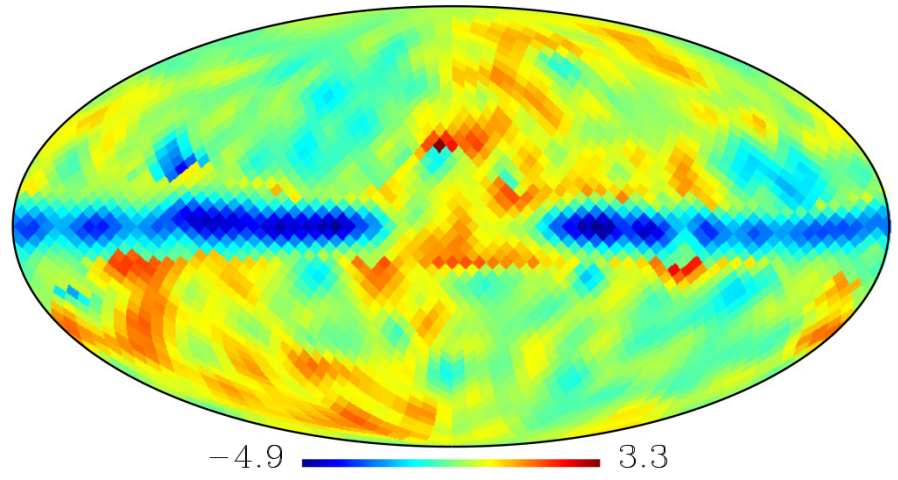

FIG. 4.- Difference of full sky cleaned CMB maps (CMap2-CMap1) obtained from Method 1 and Method 2. CMap2 appears to have lesser foreground contamination along the both sides of the galactic plane. Temperature scale is in $\mu K$ thermodynamic unit.

2. We use this generalized inverse of the partial sky CMB covariance matrix in Eqn. 4 to obtain elements of the partial sky $\tilde{\mathbf{A}}$ matrix. Using this partial sky matrix in Eqn. 5 we obtain the weights corresponding to the ThDust 5000 sky region. Using these weights we obtain the cleaned ThDust 5000 sky region.

3. Now we replace the ThDust 5000 sky region of all foreground contaminated input maps by the cleaned region obtained above. The resulting 12 maps have their galactic regions yet to be cleaned and strongly contaminated by the foregrounds. To clean the galactic region we repeat steps 1 and 2 above over the full sky. The cleaned map obtained at this point is the full-sky cleaned map obtained by Method- 2 .

\section{RESULTS}

\subsection{Cleaned Maps}

Using the first method the weights for different WMAP and Planck channels become $-0.093,0.226,0.424,-0.392$, $-0.859,-0.105,0.195,0.390,0.890,0.906,-0.607,0.0245$ in the increasing order of frequency of the 12 input maps from 23 to $353 \mathrm{GHz}$. We use these weights to linearly combine the 12 input maps to estimate the cleaned CMB map at $N_{\text {side }}=16$ and at Gaussian beam resolution of FWHM $=9^{\circ}$ (henceforth we call this cleaned map CMap1). We show the CMap1 in the top panel of Fig. 3. Visually the CMap1 does not contain any foreground residuals. We compare this map with other foreground minimized CMB maps each of which is obtained by employing a different algorithm at higher beam and pixel resolutions, as reported in the literature. COMMANDER CMB map was obtained following joint estimation of $\mathrm{CMB}$ and all foreground components, NILC CMB maps was obtained by employing an internal linear combination algorithm in the needlet space and SMICA CMB map was obtained by using spectral matching technique (e.g., see Planck Collaboration et al. (2016b) for detailed discussion about these maps). WMAP science team produced a CMB map by using usual ILC approach in pixel space (Hinshaw et al. 2007; Gold et al. 2011). We downgrade these high resolution maps at $N_{\text {side }}=16$ and bring them to a common beam resolution of $9^{\circ}$. We show the difference of CMap1 from resulting COMMANDER and NILC maps respectively in the middle left and right panel of Fig. 3. The lower left and right panel show differences of CMap1 from SMICA and WMAP ILC maps. Since monopole and dipoles are not of any cosmological interests we have removed any residual
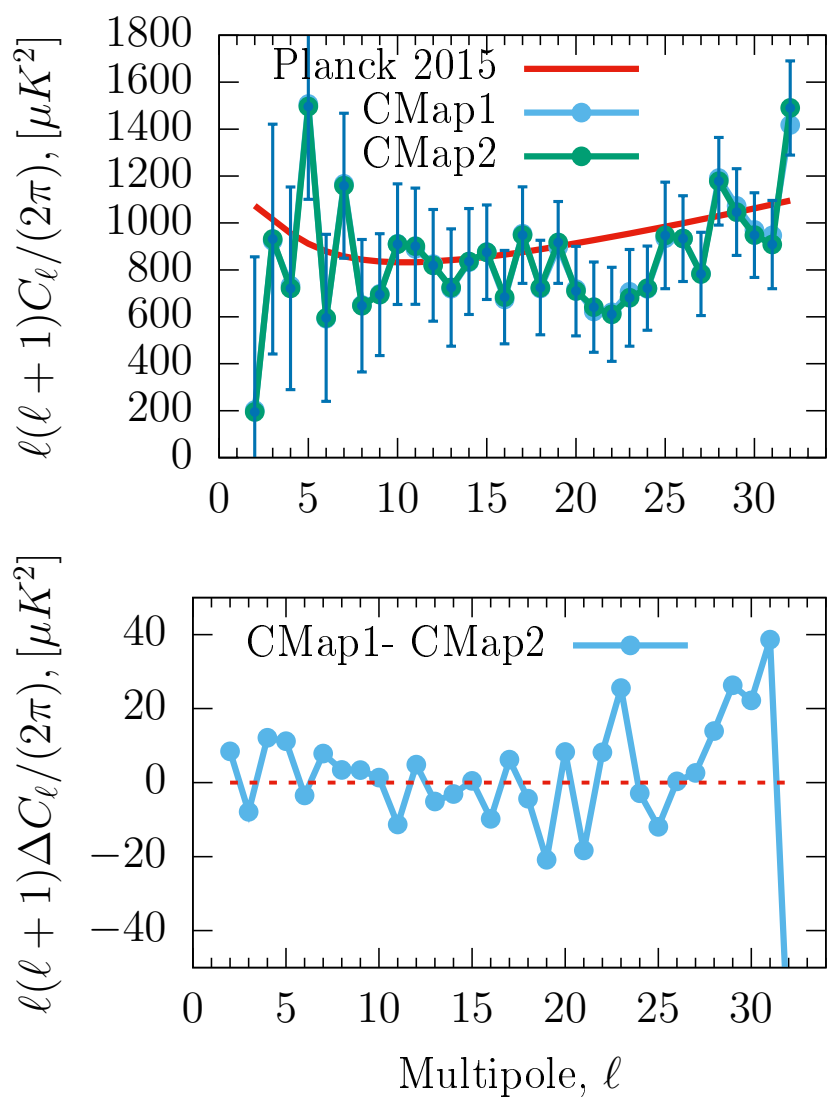

FIG. 5.- Top panel shows estimates of CMB angular power spectrum obtained from full sky region of CMap1 (Method-1), CMap2 (Method-2) along with the Planck 2015 theoretical LCDM power spectrum. Both these observed spectra of Method-1 and Method- 2 match well with each other. The error-bars are compatible to Method-2. The bottom panel shows difference of spectra obtained from these two methods. The dashed line shows the zero level of the power spectrum.

dipole and monopole from all the four difference maps shown in this figure. Clearly our cleaned CMB map matches well with these cleaned $\mathrm{CMB}$ maps in the higher galactic plane. Along the galactic plane we find some differences. However, as one can easily make out such difference along the galactic plane exists for any pair of all five low resolution CMB maps discussed in this section.

Following the second method we recover a cleaned map (CMap2) similar to CMap1. The weights for the sky region survived after application of ThDust 5000 mask are -0.066 , $0.083,0.500,-0.306,-0.562,-0.757,0.021,0.917,0.876$, $0.948,-0.684$ and 0.031 respectively for different frequencies increasing from 23 to $353 \mathrm{GHz}$ (e.g., see step 2 of Section 5.3.2). The corresponding weights for the full sky (step 3 of Method-2) linear combination are $-0.084,0.240,0.414$, $-0.399,-0.994,0.100,0.217,0.277,0.913,0.896,-0.604$ and 0.024 respectively. A common feature of the weights for both these regions is that strongly contaminated frequency maps (e.g., K1 band or $353 \mathrm{GHz}$ ) get low (negative or positive) weights to cancel out foregrounds from all frequencies. The CMap2 matches closely with the CMap1. We show the difference CMap2 - CMap1 in Fig. 4. Clearly the Method2 has slightly less foreground residuals along the both sides of the galactic plane at the expense of some additional detector noise residuals along the ecliptic plane. We compare the full sky power spectra of CMap1 and CMap2 along with other 

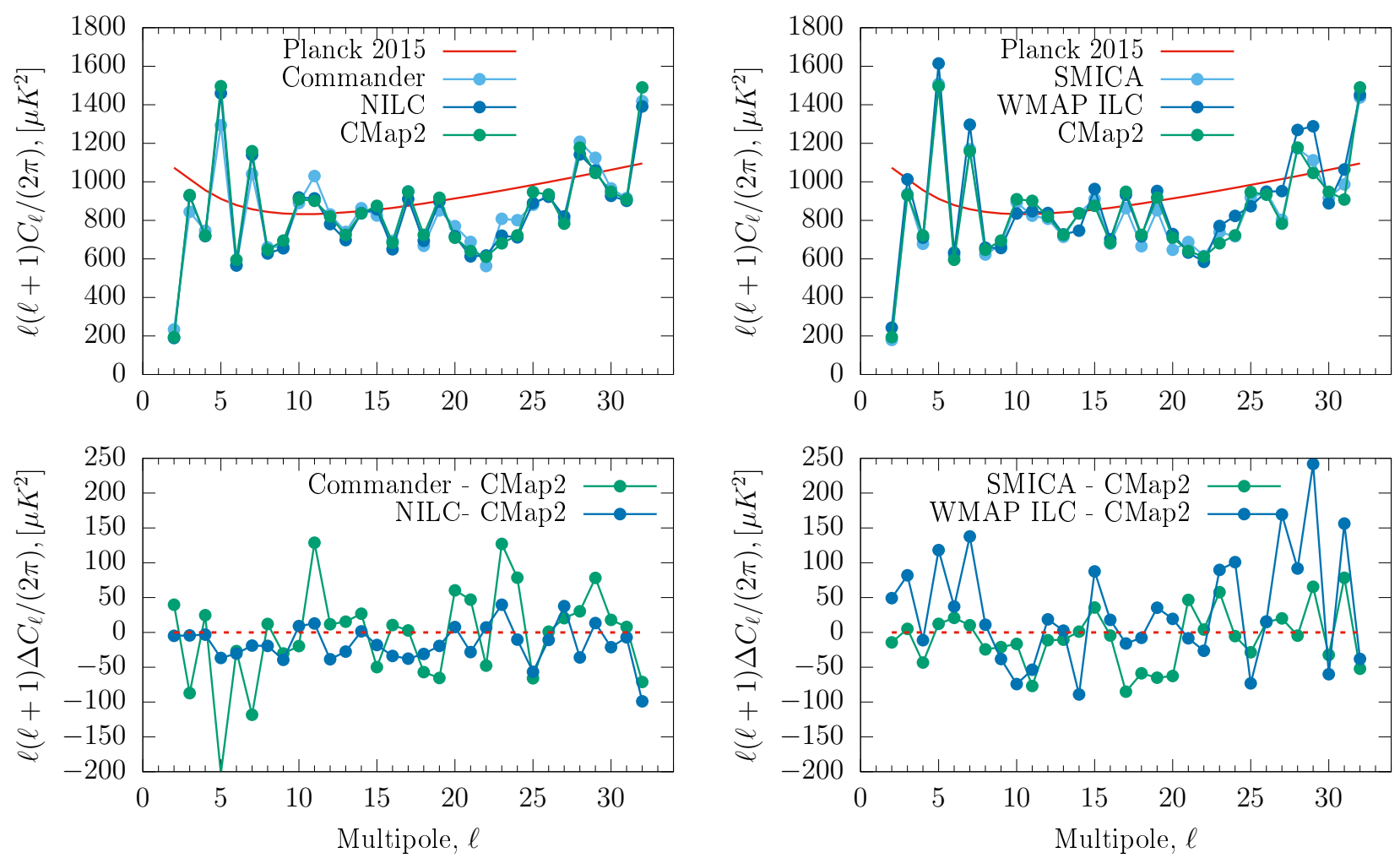

FIG. 6.- Both figures of top panel shows CMB angular power spectrum obtained from CMap2 of this work with the same estimated from other foreground cleaned CMB maps as mentioned. The bottom panel shows the difference of CMap2 spectrum with those obtained from the other foreground cleaned maps of the top panel. The zero level is shown by the red-dashed line.

CMB spectra in Section 6.2.

\subsection{Power Spectrum}

We show the CMB angular power spectra after corrections of beam and pixel effects obtained from full sky of CMap1 and CMap2 in the top panel of Fig. 5. The theoretical CMB angular power spectrum is shown in red line to guide the eye. The error-bars show the reconstruction error in power spectrum obtained from Method-2 and agree well with the cosmic variance induced errors (e.g., see Section 7). The bottom panel of this figure show difference of the spectra of these two maps. As we see from this figure both spectra match very well with each other. Such close match is also expected from the very small difference between the two cleaned maps as shown in Fig. 4. This results suggest that our new ILC approach is very weakly dependent on the sky divisions. This justifies following a global approach of foreground cleaning on large angular scales on the sky, as is done in this work. However, since method 2 simultaneously follows a global approach and performs foreground removal in an iterative fashion, we treat $\mathrm{CMB}$ angular power spectrum of $\mathrm{CMap} 2$ as the main power spectrum of this work estimated using low resolution Planck and WMAP maps.

We compare full sky CMB angular power spectrum obtained from CMap2 with the corresponding spectra obtained from COMMANDER, NILC, SMICA and WMAP ILC maps. We show these spectra in top left and top right panels of Fig. 6. Also shown in these two panels is CMB theoretical angular power spectrum obtained from Planck 2015 results. The bottom panels of this figure show the difference of angular power spectra of this work with the other spectra of the corresponding top panels. As we see from this figure the $\mathrm{CMB}$ angular power spectrum from CMap2 match closely with the angular spectra of these cleaned maps. A similar result was obtained considering CMB angular power spectrum from CMap 1 also. It is noteworthy that power spectra of CMap2 and NILC map agree excellently for the entire multipole range $2 \leq \ell \leq 32$.

\section{MONTE CARLO SIMULATIONS}

\subsection{Input CMB, Foreground and Noise Maps}

We validate the methodology for the first and second methods by performing detailed Monte Carlo simulations of the entire foreground removal and power spectrum estimation procedures. For this purpose we first generate foreground maps at different WMAP and Planck frequency bands of this work. The free free, synchrotron and thermal dust emissions at different frequencies are first obtained at $N_{\text {side }}=256$ and beam resolution $1^{\circ}$ following the procedure as described in Sudevan et al. (2017) ${ }^{3}$. We then downgrade the pixel resolution of each component map to $N_{\text {side }}=16$ and smooth each one by Gaussian beam function of FWHM $=\sqrt{540^{2}-60^{2}}=$ $536.66^{\prime}$ so as to bring all component maps for all frequency maps to the common resolution of $9^{\circ}$. We generate $\mathrm{CMB}$ temperature anisotropy maps at $N_{\text {side }}=16$ and $\mathrm{FWHM}=9^{\circ}$ by using the theoretical CMB power spectrum consistent with cosmological parameters obtained by Planck Collaboration et al. (2016d). The procedure to generate the detector noise maps remains similar to Sudevan et al. (2017). Following the same procedure as described by these authors, we first generate noise maps at $N_{\text {side }}=512$ (for WMAP DA maps) or 1024 and 2048 (for Planck frequency maps). We then convert these

\footnotetext{
${ }^{3}$ Unlike the work of Sudevan et al. (2017) in the current work we use a spatially constant spectral index $\left(\beta_{s}=-3.00\right)$ for synchrotron component for all WMAP and Planck frequencies.
} 


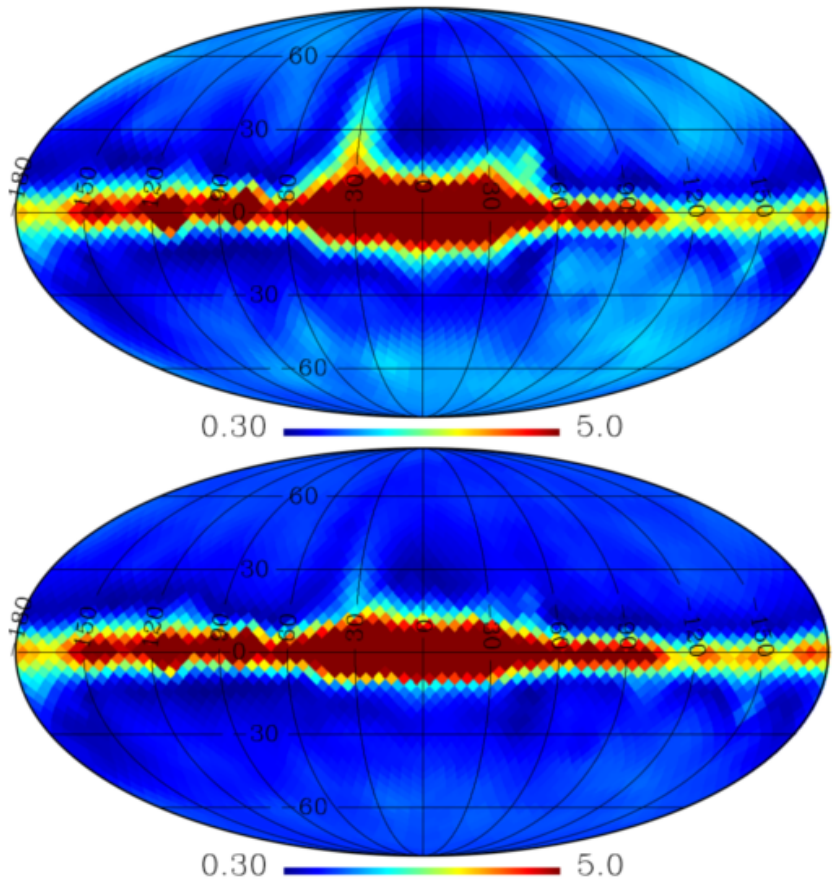

FIG. 7.- Top panel shows standard deviation map obtained from the difference of foreground minimized CMB map and corresponding randomly generated input CMB map using 200 Monte Carlo simulations of foreground minimization following Method-1 as described in Section 5.2. The bottom panel shows the standard deviation map obtained for the 200 Monte Carlo simulations of Method-2 (e.g., see Section 5.3). All units are in $\mu K$ thermodynamic temperature. The reduction in reconstruction error for these two methods is discussed in Section 7.2.

maps to spherical harmonic space upto $\ell_{\max }=32$, and multiply the resulting spherical harmonic coefficients by the ratio of the window function corresponding to FWHM $=9^{\circ}$ and the native beam window function of each WMAP DA (or Planck frequency bands). For WMAP Q, V and W band each, we average the DA noise maps to generate a single noise map corresponding to the given frequency band. We generate a set of 200 noise maps for each of 12 frequency maps of our analysis. Each of these noise maps have uncorrelated noise properties. We add the CMB, foreground and noise maps generated above to obtain a set of frequency maps that represent realistic observations of WMAP and Planck missions at $N_{\text {side }}=16$ and $\mathrm{FWHM}=9^{\circ}$. We generate a total of 200 such sets of input frequency maps for Monte Carlo simulations.

\subsection{Results}

\subsubsection{Reconstruction Error in Cleaned Maps}

If the input $\mathrm{CMB}$ map for the $i$ th Monte Carlo simulations is denoted by $T_{i}(p)$, where $p$ denotes the pixel index, and the corresponding foreground minimized CMB map is $T_{i}^{\prime}(p)$, the map representing reconstruction error for the particular simulation is then given by $\Delta T_{i}(p)=T_{i}^{\prime}(p)-T_{i}(p)$. We estimate the standard deviation map using all 200 error maps for each of our two methods of this work. The error-maps for method 1 and 2 are shown respectively is top and bottom panels of Fig. 7. As seen from this figure, using the iterative method reduces the reconstruction error in the north and southern hemisphere towards the galactic center region. Also seen from this figure is lower reconstruction error near the north polar spur region. The average variance per pixel over full sky for method 1 (estimated from the top panel Fig. 7) is $6.41 \mu K^{2}$ compared to a value of $5.25 \mu K^{2}$ for the method 2 (bottom
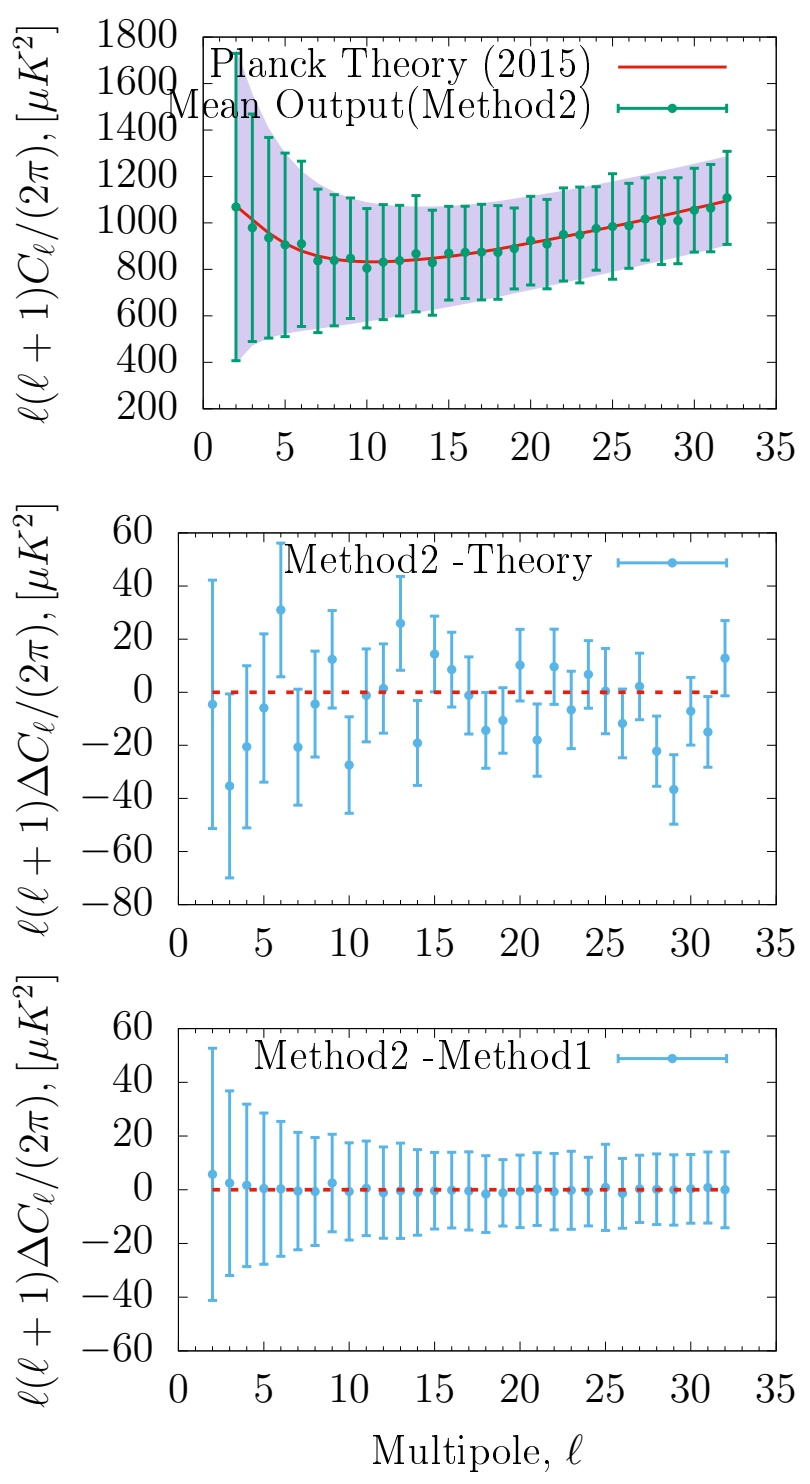

FIG. 8.- Top panel shows the mean (in green) of 200 full sky CMB angular power spectra obtained from Monte Carlo simulations of Method-2 of this work along with the theoretical CMB power spectrum (red line). The error bar computed from cosmic variance estimated from the theoretical power spectrum shown by the filled region. The reconstruction error on cleaned $\mathrm{CMB}$ power spectrum obtained from any one of the simulations is shown in green. The middle panel shows a close comparison of mean CMB angular power spectrum following Method-2 and theoretical CMB power spectrum. The error-bars of this plot represents error on the foreground cleaned mean CMB spectrum. The bottom panel shows the difference between the mean spectra of Method-2 and Method-1 along with the error bars applicable for mean spectrum of Method-1.

panel). Corresponding average variances for ThDust 5000 mask region are 1.75 and $0.97 \mu K^{2}$ respectively. For galactic region not covered by the thermal dust mask the average variances become 22.84 and $20.36 \mu K^{2}$ respectively for the Method-1 and Method-2. We conclude both methods work with comparable efficiencies, however, the second method performs better than the first method in terms of foreground removal.

\subsection{2. $C M B$ Angular Power Spectrum}

Using 200 foreground cleaned maps obtained from Monte Carlo simulations of foreground removal and subsequent 
CMB angular power spectrum estimation over the complete sky region we assess reconstruction error in cleaned CMB power spectra obtain using Method-1 and Method-2. In top panel of Fig. 8 we plot mean CMB angular power spectrum (green points) obtained following Method-2 along with the standard deviation of the cleaned power spectrum for any one of the simulations. The mean foreground cleaned power spectrum agrees well with the theoretical CMB power spectrum (red line) which is used to generate random (and isotropic) CMB realizations. The cosmic variance error limit is shown by the colored band around the theoretical CMB power spectrum. The close match of cosmic variance and the reconstruction error on the cleaned power spectrum at each multipole implies that the recovered angular power spectrum is only cosmic variance limited and reconstruction error due to foreground residuals (plus any error induced by detector noise) is a sub-dominant source of contamination on the angular scale chosen in this work. In the middle panel of Fig. 8 we closely investigate any reconstruction biases that may exist in the foreground cleaned power spectrum of Method- 2 by plotting the difference between foreground minimized mean $\mathrm{CMB}$ power spectrum and the CMB theoretical power spectrum. The error bar at each multipole plotted in this panel is applicable for the mean CMB angular power spectrum and therefore they are obtained by scaling the corresponding reconstruction error of top panel by $1 / \sqrt{N_{\text {sim }}}$ where the number of simulations, $N_{\text {sim }}=200$. For all the multipoles except $(\ell=29)$ the significance of any difference between the mean cleaned spectrum and the theoretical CMB spectrum is less than $2 \sigma$. For $\ell=29$ the significance of deviation is $2.8 \sigma$. This shows that power spectrum obtained from Method- 2 has no significant bias that may arise due to imperfect foreground residuals. The bottom panel of Fig. 8 shows the difference between mean CMB power spectra obtained from Method-1 and Method-2. The error-bars of this plot is computed from foreground cleaned maps of Method-1 and they are applicable for the mean power spectrum. Clearly mean spectra obtained by the two methods of this work agree very well with each other. Both methods produce comparable error-bars as well.

\section{ADVANTAGE OF GLOBAL ILC METHOD AT LOW RESOLUTION}

The global ILC method has two very important advantages over the usual ILC method in pixel space that does not take into account prior information about $\mathrm{CMB}$ theoretical covariance matrix. First, the globally cleaned CMB map has less reconstruction error at each pixel. Second, the usual ILC approach (without using the covariance information) at low resolution leads to a bias in the power spectrum which remains absent in the proposed methods of this work. The cause of these limitations in usual ILC approach at low resolution analysis is a chance-correlation between the CMB and foreground (and detector noise) components which can not be ignored over large scales of the sky. In this section we discuss about the advantages of our approach.

Using the simulated frequency maps at $N_{\text {side }}=16$ and $9^{\circ}$ resolution (e.g., Section 7.1) we perform 200 Monte Carlo simulations over the complete sky region using usual ILC approach, wherein no CMB covariance matrix is used. The error map in $\mathrm{CMB}$ reconstruction is then computed in the same fashion as discussed in Section 7.2.1. The standard deviation map is plotted in Fig. 9 which indicates a strong residual, not only on the the galactic plane, but also in higher galactic latitudes. Unlike the small variance per pixel reported in Section 7.2.1 the average variance per pixel for Fig. 9 is large

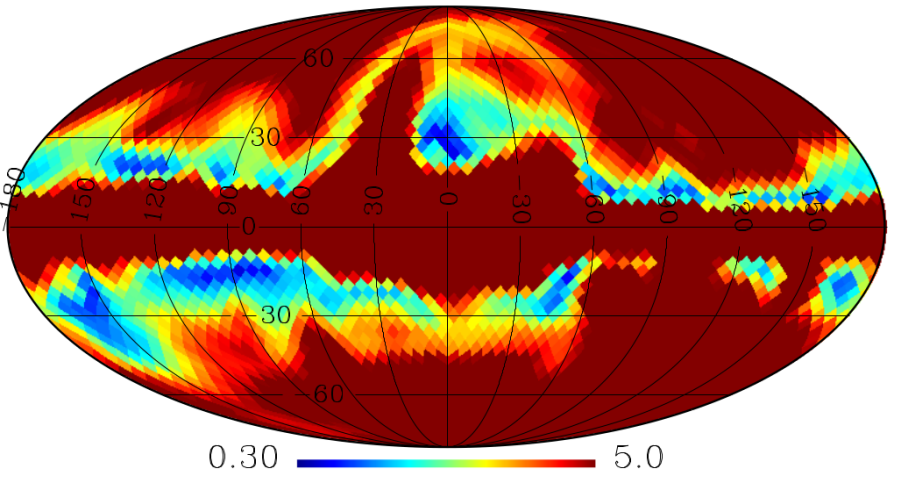

FIG. 9.- The standard deviation map indicating the reconstruction error for usual pixel space ILC approach over the entire sky at $N_{\text {side }}=16$ and $9^{\circ}$ resolution. Large reconstruction error compared to the methods (e.g., see Fig. 7) of this work is seen. Unit is in $\mu K$ thermodynamic.
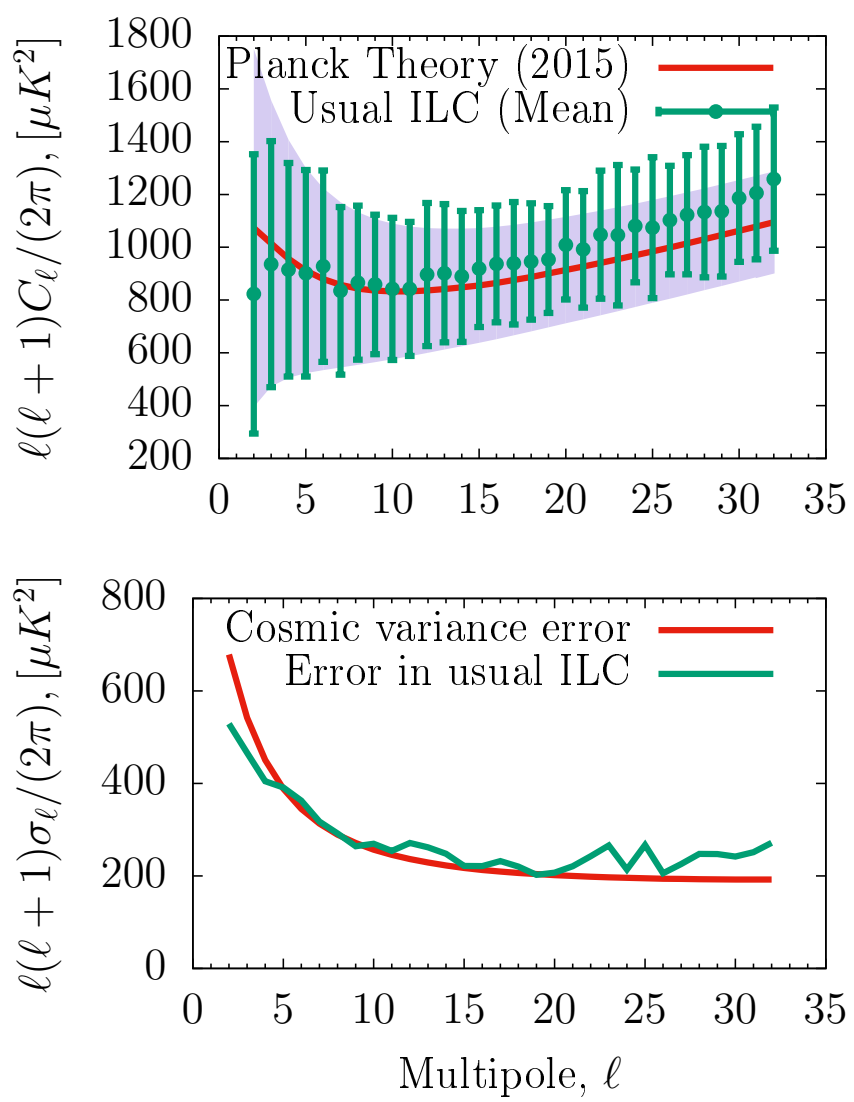

FIG. 10.- Top panel shows the mean CMB power spectrum (in green) obtained from 200 Monte Carlo simulations of usual ILC approach over the entire sky on low resolution maps as discussed in Section 8 along with the theoretical CMB angular power spectrum (red line) consistent with Planck 2015 results. The filled color band shows the cosmic variance excursion limit of the observed CMB angular power spectrum. The green error-bars show reconstruction error in the cleaned power spectrum at different multipoles. The bottom panel closely compares the reconstruction error-bars with the cosmic variance induced errors. Residuals in the cleaned maps cause larger than cosmic variance error starting from $\ell \sim 10$. For low multipoles, $\ell \leq 4$ reconstruction error becomes less than cosmic variance induced error since the cleaned power spectrum at low multipoles is biased low due to a chance correlation of CMB with foregrounds (and detector noise).

$\left(89.17 \mu K^{2}\right)$. This clearly demonstrates the first advantage, i.e., sharp decrease in reconstruction error of cleaned CMB map, when we incorporate prior information about theoretical covariance of CMB component. 


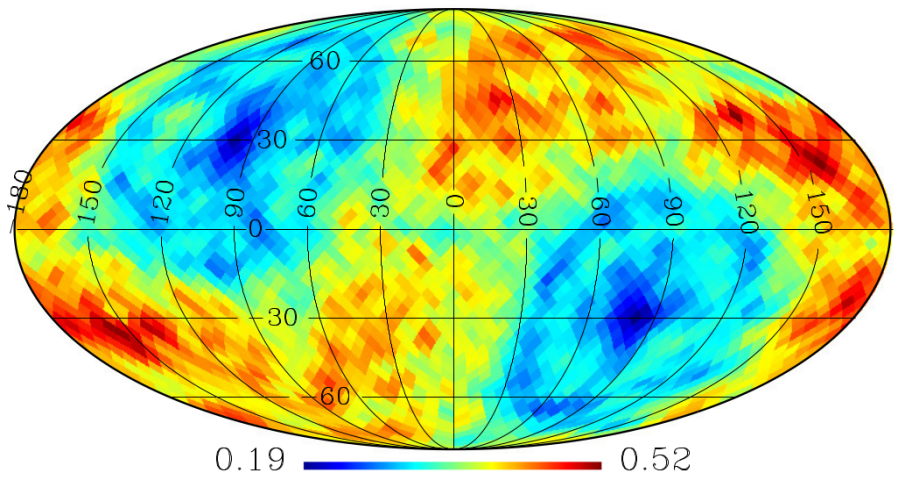

FIG. 11.- The standard deviation map computed from the difference of cleaned CMB maps and corresponding input CMB maps using ILC method, when the weights are obtained from Eqn. 12. The reconstruction error follows a noise pattern and is much smaller compared to Fig. 9 when CMBforeground chance correlation affects the weight estimation. Unit is in $\mu K$ thermodynamic.

The larger reconstruction error in cleaned maps in usual ILC approach, causes a significant bias in the power spectrum which is a quadratic function of the data. We show the mean power spectrum computed from 200 Monte Carlo simulations of usual ILC approach over the entire sky in green in top panel of Fig. 10 along with the Planck 2015 theoretical power spectrum which is used to generate the input CMB maps. Clearly a positive bias exist due to imperfect foreground residuals in the cleaned spectrum starting from multipole $\ell=8$. Another interesting feature of the top panel is existence of a negative bias for $\ell \leq 5$. Such negative bias is expected and was first reported by Saha et al. (2006) and is discussed extensively in Saha et al. (2008) (see also Sudevan et al. (2017) for such bias in high resolution analysis) for multipole space ILC methods. In fact, observing the error pattern of Fig. 9 it is likely that a positive bias due to residual foregrounds exists even at low multipoles, $\ell \leq 8$ on the top of the additional negative bias in this multipole range. The bottom panel of Fig. 10 compares the reconstruction error in the cleaned power spectrum with the error due to cosmic variance alone. Starting from multipole $\ell \sim 10$ we see that the error in usual ILC power spectrum becomes larger than the cosmic variance induced error. Interestingly, due to existence of negative bias at the low multipoles the error in cleaned spectrum become biased low for $\ell \leq 4$. The bias existing in the cleaned power spectrum of the usual ILC approach at low resolution along with larger error in reconstructed power spectrum from this approach justifies our second point of advantage (discussed at the beginning of the current section) of the new approach described in this article.

\section{ROLE OF CMB-FOREGROUND (OR CMB-NOISE) CHANCE CORRELATION}

Having discussed in the previous section the advantages of the global ILC method of this work we now focus on the cause of excess residuals in the usual ILC method when applied to low resolution maps. If we apply usual ILC method on the input maps described in Section 2 variance of the cleaned map becomes,

$$
\hat{\sigma}^{2}=\mathbf{W} \hat{\mathbf{C}} \mathbf{W}^{T},
$$

where $\hat{\mathbf{C}}$ is an $n \times n$ matrix representing the covariance between different input frequency maps (from which mean temperature anisotropies corresponding to each frequency is already subtracted). Similar to Eqn. 5 the set of weights that minimizes variance of the cleaned map subject to the con- straint CMB is preserved is given by (Saha et al. 2008; Tegmark et al. 2003; Tegmark \& Efstathiou 1996),

$$
\mathbf{W}=\frac{\mathbf{e} \hat{\mathbf{C}}^{\dagger}}{\mathbf{e}^{\dagger} \mathbf{e}^{T}} .
$$

The data covariance matrix $\hat{\mathbf{C}}$ follows, $\hat{\mathbf{C}}=\hat{\sigma}_{c}^{2} \mathbf{e}^{T} \mathbf{e}+\hat{\mathbf{C}}_{f c}+\mathbf{C}_{f}$ where $\hat{\sigma}_{c}^{2}$ represents the variance of the CMB component which is independent on the frequency, $\hat{\mathbf{C}}_{f c}$ is an $n \times n$ matrix denoting the chance correlation between the $\mathrm{CMB}$ and all foreground components for a given realization of $\mathrm{CMB}$ (e.g., pure CMB signal in our Universe) and finally $\mathbf{C}_{f}$ is the $n \times n$ foreground covariance matrix ${ }^{4}$. Following Saha et al. (2008) we note that, $\mathbf{e}^{T} \in \mathcal{C}\left(\hat{\mathbf{C}}_{f c}+\mathbf{C}_{f}\right)$ so that the generalized Sherman-Morrison formula for Moore-Penrose generalized inverse of rank one update becomes,

$$
\hat{\mathbf{C}}^{\dagger}=\hat{\mathbf{A}}^{\dagger}-\frac{1}{\lambda} \mathbf{f g}^{T}
$$

where $\hat{\mathbf{A}}=\hat{\mathbf{C}}_{f c}+\mathbf{C}_{f}, \lambda=1+\mathbf{e} \hat{\mathbf{A}}^{\dagger} \mathbf{e}^{T}, \mathbf{f}=\hat{\mathbf{A}}^{\dagger} \mathbf{e}^{T}$ and $\mathbf{g}=\hat{\mathbf{A}}^{\dagger} \mathbf{e}^{T}$. Using Eqn. 10 we obtain,

$$
\mathbf{W}=\frac{\mathbf{e} \hat{\mathbf{A}}^{\dagger}}{\mathbf{e}^{\dagger} \mathbf{e}^{T}} .
$$

Using Eqn. 11 we conclude that the weights are independent on the exact level of CMB variance $\hat{\sigma}_{c}^{2}$ for the particular random realization. This is expected since the weights in the usual ILC method, in principle, should only be determined by the foregrounds as long as CMB follows blackbody distribution. One may interpret Eqn. 11 as the usual ILC weights minimizing the part of the variance in the cleaned map that arise due to CMB-foreground chance correlation and foreground components. Since $\hat{\mathbf{A}}=\hat{\mathbf{C}}_{f c}+\mathbf{C}_{f}$, we see from Eqn. 11 that in practice the ILC weights not only depend on foreground covariance matrix $\mathbf{C}_{f}$ but also they depend upon the CMBforeground chance correlation matrix $\hat{\mathbf{C}}_{f c}$. What will happen if in Eqn. 11 we could replace $\hat{\mathbf{A}}$ by $\mathbf{C}_{f}$ ? We note that such a choice is not possible for analysis of the real data since the covariance matrix for the foregrounds is not known exactly a priori. However, in Monte Carlo simulations we can always assume that $\mathbf{C}_{f}$ is known. This will be the situation when weights are not affected by the chance-correlation matrix. If we know the true foreground covariance matrix accurately, in usual ILC procedure one will just minimize the part of the variance in the cleaned map that arise due to foreground components. Clearly this is $\sigma_{f}^{2}=\mathbf{W} \mathbf{C}_{f} \mathbf{W}^{T}$. Minimizing $\sigma_{f}^{2}$ subject to the constraint $\mathrm{CMB}$ is preserved gives,

$$
\mathbf{W}=\frac{\mathbf{e} \mathbf{C}_{f}^{\dagger}}{\mathbf{e C}_{f}^{\dagger} \mathbf{e}^{T}} .
$$

We perform detailed Monte Carlo simulations of foreground minimization at low resolution following usual ILC method, with simulated WMAP and Planck observations to investigate the difference in the cleaned maps obtained by two different

\footnotetext{
${ }^{4}$ In this discussions we have assumed that the detector noise contribution is small compared to the foreground or CMB signal. This is the case for WMAP and Planck temperature observation over the large scales of the sky. We emphasize that we do not require detector noise to be completely absent, we only assume that the data signal dominated. Accordingly, we interpret $\mathbf{C}_{f}$ to contain a small amount of detector noise also
} 

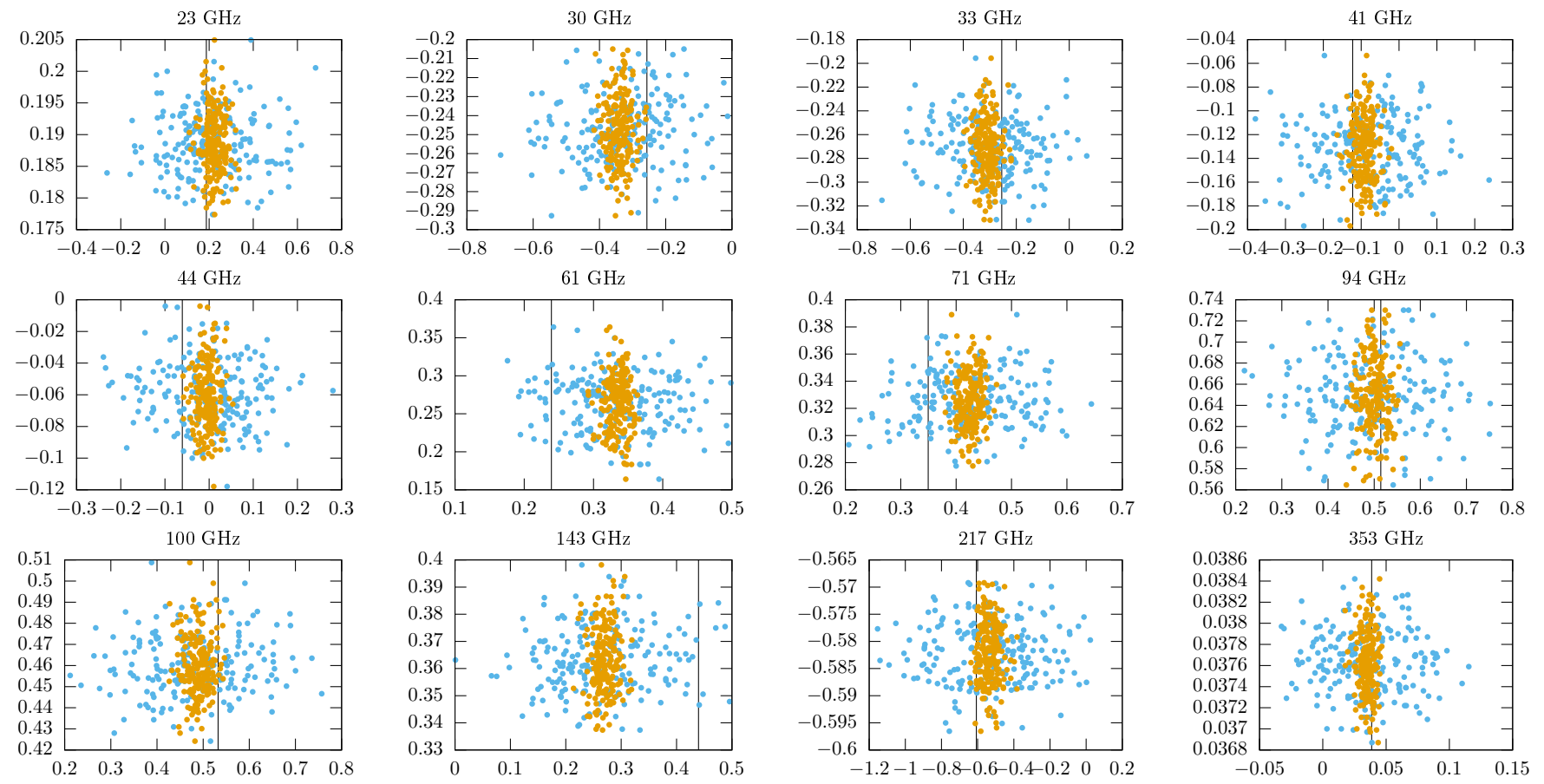

FIG. 12.- Scatter plot obtained from Monte Carlo simulations showing lower dispersion (along horizontal axis) of weights (yellow) when we follow global ILC method with prior information from theoretical CMB covariance matrix on large scales on the sky for different WMAP and Planck frequency bands. The y coordinates of blue or yellow points represent weights obtained from Monte Carlo simulations using Eqn. 12. The larger dispersion of blue points along the horizontal axes causes larger reconstruction error in cleaned maps for usual ILC methods at low resolution. The vertical lines represent values of weights obtained using Eqn. 12 but without any detector noise in the simulations.

ways. First, the weights are determined following Eqn. 11 and second, they are determined by Eqn. 12. In the first case we recover results that are similar to those shown in Figs. 9 and 10. This implies in presence of CMB-foregrounds chance correlations usual ILC method perform a poor foreground subtraction on large scales on the sky. In the second case, when the chance correlation matrix is absent the method performs foreground removal very well. The standard deviation map computed from the difference of cleaned CMB maps and the corresponding input CMB maps, for this case, is shown in Fig. 11. The standard deviation map is consistent with a detector noise pattern without any visible signature of residual foregrounds. The mean pixel variance of this map is only $0.14 \mu K^{2}$ indicating greatly improved foreground subtraction compared to the case when CMB-foreground chance correlation is present. We reemphasize that, although, we can use Eqn. 12 for the case of Monte Carlo simulations where the input foreground models are known, in practice, we can not use this equation to estimate ILC weights since the foreground covariance matrix $\mathbf{C}_{f}$ is unknown for the observed sky. We use Eqns. 12 and 11 in Monte Carlo simulations to establish that the CMB-foreground chance correlations cause significant residuals in usual ILC method. The global ILC method that propose to use CMB covariance information, thus, becomes greatly beneficial method, improving performance of usual ILC method without any need to know $\mathbf{C}_{f}$.

Apart from comparing the pixel reconstruction error maps (e.g., Figs. 9 and 11) or the power spectra of cleaned maps there is another way in which we see that using the theoretical CMB covariance matrix helps to greatly improve usual ILC results. In Fig. $12(x, y)$ coordinates of any blue point are given respectively by value of weight for a particular frequency band obtained using the usual ILC method and the corresponding value of the weight using Eqn. 12 while clean- ing a given set of input frequency maps. The $y$-coordinate of the yellow points are same as the blue point for the same set of input frequency maps, however the $x$-coordinate of yellow points represents weights for the global ILC method using information about the theoretical CMB covariance matrix. The blue points show significantly larger dispersion along the horizontal axes for all frequency bands compared to the corresponding dispersion of yellow points. The new method of this work efficiently reduces the larger dispersion of weights of usual ILC method and produces better foreground minimized CMB maps at low resolution. The $y$-coordinates of all points of this figure show some level of fluctuations, even if we use Eqn. 12 to estimate the weights that represent the $y$ coordinates. This is because apart from the foregrounds $\mathbf{C}_{f}$ contain a small level of detector noise. The $x$-coordinate of vertical axis of each plot show the value of the weight when no detector noise is present in $\mathbf{C}_{f}$. Each of these values remains same for different Monte Carlo simulations and represent weights that will be necessary to remove foregrounds in an ideal noise-less experiment. We finally note that using CMB theoretical covariance matrix in Eqn. 2 we efficiently suppress CMB large angle covariances which leads to significantly smaller dispersion of weights because of smaller CMBforeground chance correlation. The small dispersion of our weights results in a greatly improved foreground minimization than the usual ILC method on large scales of the sky.

\section{DISCUSSIONS \& CONCLUSION}

We have developed a new ILC method for foreground minimization in pixel space for application on large angular scales on the sky using prior information about theoretical CMB covariance matrix. We apply the methodology on low resolution WMAP and Planck frequency maps and show that the cleaned CMB temperature anisotropy map obtained by us 
match very well with those obtained by other science groups of Planck and WMAP. This shows that results of CMB maps and its power spectrum are robust with respective to a variety of analysis pipeline. We validate the methodology of our foreground removal by detailed Monte Carlo simulations. Usage of this new approach has several benefits over naive application of usual ILC approach in pixel space over large scales of the sky.

1. First, the new approach generates cleaned CMB map that has significantly lower reconstruction error due to foreground residuals. The power spectrum from the cleaned map also has the lower reconstruction error for our case, the standard deviations of $\mathrm{CMB}$ angular power spectrum estimated from the Monte-Carlo simulations agree with those estimated from the cosmic variance alone.

2. Second, the CMB angular power spectrum obtained from our cleaned maps does not have any visible signature of negative bias at the low multipole region, which is seen to be present for pixel space application of usual ILC method over large scales on the sky. Such negative bias is also reported in harmonic space ILC method by Saha et al. (2006) and its property and origin were investigated in detail by Saha et al. (2008). The negative bias arise due a chance correlation between CMB and foreground components on a particular realizations of the sky. Using inverse weight of CMB theoretical covariance matrix in Eqn. 2 we effectively get rid of such chance correlations and the as well as the resulting negative biases in the cleaned CMB angular power spectrum at low multipoles.
The new method complements the usual ILC approach in pixel space which so far has been applied on high resolution maps by incorporating local information available from input frequency maps to better remove foregrounds, the spectral property of which vary with the sky positions. On the very large scales the spectral properties of foregrounds are expected to vary by small amount over the entire sky. We show that, on the large scale it is sufficient to perform ILC foreground removal by dividing the sky merely into two regions, provided we use the prior information available from CMB covariance matrix globally on the sky. Although we have assumed a theoretical CMB covariance matrix consistent with assumption of statistical isotropy of CMB in Eqn. 6, in principle, one can also use a covariance matrix in our method which is not statistically isotropic. This brings about a possibility to open up a new avenue to incorporate such additional information in our method which may be a signature of non trivial primordial power spectrum (Ghosh et al. 2016; Contreras et al. 2017). Taking into account the global nature of our low resolution analysis and local nature of high resolution analysis of usual ILC method, we now consider pixel space ILC method in a general perspective that incorporates a very comprehensive duality in its nature. We hope that our method will be useful to analyze low resolution polarization maps from Planck or future generation CMB missions.

We use publicly available HEALPix Górski et al. (2005) package available from http://healpix.sourceforge.net for some of the analysis of this work. We acknowledge the use of Planck Legacy Archive (PLA) and the Legacy Archive for Microwave Background Data Analysis (LAMBDA). LAMBDA is a part of the High Energy Astrophysics Science Archive Center (HEASARC). HEASARC/LAMBDA is supported by the Astrophysics Science Division at the NASA Goddard Space Flight Center.

\section{REFERENCES}

Basak, S., \& Delabrouille, J. 2012, MNRAS, 419, 1163

-. 2013, MNRAS, 435, 18

Bennett, C. L., Hill, R. S., Hinshaw, G., et al. 2003, ApJS, 148, 97

Bouchet, F. R., Prunet, S., \& Sethi, S. K. 1999, MNRAS, 302, 663

Bunn, E. F., Fisher, K. B., Hoffman, Y., et al. 1994, ApJ, 432, L75

Contreras, D., Zibin, J. P., Scott, D., Banday, A. J., \& Górski, K. M. 2017, Phys. Rev. D, 96, 123522

Eriksen, H. K., Banday, A. J., Górski, K. M., \& Lilje, P. B. 2004, ApJ, 612, 633

Eriksen, H. K., Dickinson, C., Jewell, J. B., et al. 2008a, ApJ, 672, L87

Eriksen, H. K., Jewell, J. B., Dickinson, C., et al. 2008b, ApJ, 676, 10

Eriksen, H. K., Huey, G., Saha, R., et al. 2007, ApJ, 656, 641

Fixsen, D. J., Cheng, E. S., Gales, J. M., et al. 1996, ApJ, 473, 576

Ghosh, S., Kothari, R., Jain, P., \& Rath, P. K. 2016, J. Cosmology Astropart. Phys., 1,046

Gold, B., Odegard, N., Weiland, J. L., et al. 2011, ApJS, 192, 15

Górski, K. M., Hivon, E., Banday, A. J., et al. 2005, ApJ, 622, 759

Hinshaw, G., Nolta, M. R., Bennett, C. L., et al. 2007, Astrophys. J. Suppl. Ser., 170, 288

Mather, J. C., Cheng, E. S., Cottingham, D. A., et al. 1994, ApJ, 420, 439
Moore, E. H. 1920, Bull. Am. Math. Soc., 26, 394, unpublished address. Available at http://www.ams.org/journals/bull/1920-26-09/S0002-99041920-03322-7/S0002-9904-1920-03322-7.pdf.

Penrose, R. 1955, Mathematical Proceedings of the Cambridge Philosophical Society, 51, 406

Planck Collaboration, Adam, R., Ade, P. A. R., et al. 2016a, A\&A, 594, A9 -. 2016b, A\&A, 594, A9

-. 2016c, A\&A, 594, A10

Planck Collaboration, Ade, P. A. R., Aghanim, N., et al. 2016d, A\&A, 594, A13

Purkayastha, U., \& Saha, R. 2017, ArXiv e-prints, arXiv:1707.02008

Saha, R. 2011, ApJ, 739, L56

Saha, R., \& Aluri, P. K. 2016, ApJ, 829, 113

Saha, R., Jain, P., \& Souradeep, T. 2006, ApJ, 645, L89

Saha, R., Prunet, S., Jain, P., \& Souradeep, T. 2008, Phys. Rev. D, 78, 023003

Sudevan, V., Aluri, P. K., Yadav, S. K., Saha, R., \& Souradeep, T. 2017, ApJ, 842,62

Tegmark, M., de Oliveira-Costa, A., \& Hamilton, A. J. 2003, Phys. Rev. D, 68,123523

Tegmark, M., \& Efstathiou, G. 1996, Mon. Not. R. Astron. Soc., 281, 1297 